\title{
Éditorial
}

\section{La collaboration pluridisciplinaire}

$\mathrm{D}$ epuis la collaboration orthodontico-chirurgicale entre Edward Angle et Vilray Blair au début du XX⿳⺈冂大 siècle, la prise en charge des dysmorphoses dento-squelettiques a considérablement évoluél. On ne peut nier que le « couple orthodontiste/chirurgien » reste aujourd'hui central dans la construction et le déroulement d'un protocole orthodontico-chirurgical. Mais si l'objectif des pionniers de la chirurgie orthognathique se limitait principalement à restaurer une classe I squelettique afin de permettre à l'orthodontiste de retrouver une classe I dentaire stable, l'ambition des acteurs de la prise en charge d'une dysmorphose dento-squelettique est toute autre en 2016. Un triple objectif de restauration des fonctions orofaciales, de bon résultat esthétique et de stabilité occlusale doit être recherché et cela nécessite une excellente collaboration pluridisciplinaire².

L'objectif fonctionnel de base reste bien sûr l'obtention d'une classe I dentaire et squelettique stable, et d'un engrènement dentaire favorable à une mastication efficace et à une certaine stabilité primaire du traitement orthodontique. Mais la conscience de l'importance des fonctions oro-faciales de chaque patient dysmorphique s'est considérablement renforcée depuis une vingtaine d'années. Les dysfonctions linguales (déglutition) et respiratoires font maintenant l'objet d'une attention particulière, leur implication dans la genèse et la pérennisation des dysmorphoses faciales ayant été clairement établie. La collaboration avec nos confrères orthophonistes, ORL et kinésithérapeutes prend ici tout son sens à toutes les étapes du protocole orthodontico-chirurgical. Cette collaboration s'étend parfois jusqu'aux spécialistes du sommeil (pneumologues et $O R L$ ) chez nos patients dysmorphiques atteints d'un syndrome d'apnées hypopnées obstructives du sommeil (SAHOS). De même, la prise en charge des dysfonctions articulaires pré-et/ou post-traitement justifie l'intervention fréquente de collègues physiothérapeutes au cours du traitement et la prescription d'examens d'imagerie spécifiques tels l'IRM.

L'âge moyen des patients qui s'engagent dans un protocole orthodontico-chirurgical a nettement augmenté depuis une quinzaine d'années. La motivation souvent mise en avant chez nos patients adultes est celle de la pérennité de leur denture, pour des raisons esthétiques et fonctionnelles. Nos confrères dentistes omnipraticiens, parodontologues et implantologues ont ainsi naturellement pris une part très active dans le plan de traitement de nos patients, qui doivent impérativement être informés en tout début de traitement de l'ensemble des éléments, y compris financiers, de leur prise en charge. Une autre dimension qui est apparue et s'est développée avec la 
prise en charge de nos patients adultes est celle de l'impact psychologique de la chirurgie orthognathique. II n'est anodin pour personne de " changer de visage ", d'autant plus que ce changement est parfois subi par le patient. Cette dimension est maintenant pleinement considérée, que ce soit de manière préventive en confiant un patient ou une patiente que le praticien juge « fragile » à un confrère psychologue ou psychiatre de manière préventive, ou dans les suites de la chirurgie afin d'aider son patient à s'approprier son nouveau visage.

L'évolution vers une demande - voire une exigence - esthétique du résultat orthodontico-chirurgical est également un des moteurs puissants de l'évolution de nos protocoles. Plusieurs études ont montré que la motivation profonde, souvent non exprimée, des patients qui s'engagent dans un protocole thérapeutique orthognathique était une motivation esthétique plus que fonctionnelle3. Appréhender finement la demande esthétique faciale d'un patient, et être capable de la recentrer si cette demande est irréaliste ne sont pas choses faciles, et le résultat esthétique post-opératoire peut ne pas être parfaitement à la hauteur de l'attente du patient. C'est souvent le cas dans les asymétries faciales où les déformations de l'enveloppe tissulaire faciale ne sont que rarement parfaitement corrigées par l'équilibration dento-squelettique. Il est alors utile de confier un tel patient à un confrère chirurgien plasticien qui maîtrise les techniques de profiloplastie et de comblements sous-cutanés, et qui saura apporter les quelques améliorations esthétiques attendues par le patient.

Enfin, d'autres acteurs diagnostiques et thérapeutiques gravitent également autour du couple orthodontiste-chirurgien : le prothésiste dentaire - acteur majeur de la confection du set-up chirurgical -, les industriels de l'outillage d'ostéosynthèse qui font évoluer leur matériel au gré de demandes des chirurgiens, les radiologues qui proposent des techniques d'imagerie du massif facial toujours plus précises et moins irradiantes. Les protocoles orthodontico-chirurgicaux ne doivent se concevoir en 2016 que dans un cadre multidisciplinaire et avec un véritable esprit d'équipe.

Pr. Pierre Bouletreau, Chirurgien maxillo-facial Coordonnateur du DIU de chirurgie orthognathique CHU Lyon-Sud

\title{
RÉFÉRENCES
}

1. Steinhäuser EW. Historical development of orthognathic surgery. J Craniomaxillofac Surg 1996;24(4):195-204.

2. Bouletreau P, Raberin M. La chirurgie orthognathique est un travail d'équipe! Orthod Fr 2011;82(2):183-192.

3. Rivera SM, Hatch JP, Dolce C, Bays RA, Van Sickels JE, Rugh JD. Patients' own reasons and patient-perceived recommendations for orthognathic surgery. Am J Orthod Dentofacial Orthop 2000;118(2):134-141.

Les opinions émises n'engagent que leurs auteurs.

\author{
"L'association de la Revue d'Orthopédie Dento-Faciale \\ et le comité de rédaction \\ vous présentent leurs meilleurs vœux pour 2016 »
}

